\title{
Responsabilidad Social Corporativa Estudio sobre la identificación y clasificación de los stakeholders
}

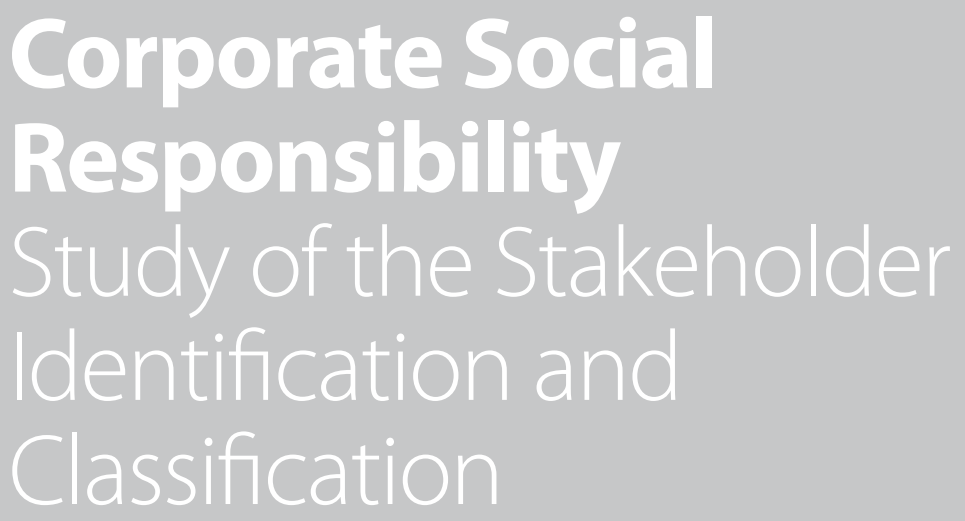

Estrella Barrio

Universidad Autónoma de Barcelona Estrella.barrio@uab.cat

Ana Ma Enrique

Universidad Autónoma de Barcelona

Anamaría.enrique@uab.cat
Barrio, E. y Enrique, A. M.a (2018)

Responsabilidad Social Corporativa. Estudio sobre la identificación y clasificación de los stakeholders Revista Internacional de Investigación en Comunicación aDResearch ESIC. № $17 \mathrm{Vol} 17$

Primer semestre, enero-junio 2018 · Págs. 90 a 109 DOI: 17.7263/ADRESIC.017.005 
RESUMEN

\section{Clasificación JEL: \\ M14}

Palabras clave:

Stakeholders,

responsabilidad

social sorporativa,

empresas,

comunicación

corporativa

Desde hace unos años estamos siendo testigos del auge de la Responsabilidad Social Corporativa (RSC) debido a una serie de fenómenos que están cambiando el panorama empresarial. Un fenómeno clave sin el que no se puede entender el apogeo de la RSC es la redefinición de la empresa desde la teoría de los stakeholders de Freeman, que concibe las organizaciones como un ente formado por diversos actores clave que afectan y son afectados por éstas. La presente investigación, basada en la metodología del estudio de caso, se centra en la identificación y tipificación de los diferentes stakeholders de una compañía internacional en materia de RSC. Para ello, el diseño de la investigación se ha basado en la técnica de la triangulación, combinando la revisión de documentos escritos con la realización de entrevistas enfocadas. Los resultados muestran que en materia de RSC los stakeholders principales de la organización están formados por los consumidores, empleados, proveedores, distribuidores, administración pública, organizaciones sin ánimo de lucro, empresas colaboradoras, medios de comunicación, comunidad local y sociedad en general. En cuanto a la catalogación de los stakeholders, a diferencia de lo detectado en la revisión de la literatura, éstos son clasificados entre beneficiarios, aquellos a los que van dirigidas las acciones de RSC (consumidores, empleados, proveedores, comunidad local y sociedad en general) y partners, aquellos que colaboran en la ejecución de las acciones de RSC, de modo que se genera una relación win-win entre ellos y la empresa (organizaciones sin ánimo de lucro, empresas colaboradoras, administración pública, distribuidores y medios de comunicación).

\section{ABSTRACT}

\section{JEL Classification: \\ M14 \\ Key words:}

Stakeholders,

Corporate Social

Responsibility,

companies,

corporate communication
In the last years, we have witnessed the rise of Corporate Social Responsibility (CSR) due to a series of phenomena that are changing the business context. One of these phenomena without which the CSR rise cannot be understood is the company's redefinition from the Freeman's stakeholder theory, which conceives organizations as entities made up of different key actors that affect and are affected by them. The present research, based on the case study methodology, focuses on an international company stakeholder identification and classification in terms of CSR. Therefore, the research design has been based on the triangulation technique, combining the review of written documents with focused interviews. The results show that in terms of CSR the main company's stakeholders are consumers, employees, suppliers, distributors, public administration, non-profit organizations, collaborating companies, media, local community and society in general. In regard to the subject of stakeholder classification, unlike what was detected in the literature review, they are classified among beneficiaries, those to whom the CSR actions are addressed to (consumers, employees, suppliers, local community and society in general) and partners, those who collaborate in CSR actions execution, so that a win-win relationship is generated between them and the company (non-profit organizations, collaborating companies, public administration, distributors and media). 


\section{Introducción}

La irrupción de los valores intangibles en el panorama empresarial ha abierto un nuevo ámbito de actuación en la Comunicación Corporativa, siendo la identidad, cultura, imagen, reputación, marca y responsabilidad social corporativa, intangibles a gestionar por los departamentos de comunicación. A las tradicionales funciones del director de comunicación como la comunicación externa e interna, gestión de la imagen y relación con los medios, se han sumado los principales intangibles (Villagra, 2015).

En el presente artículo nos centraremos en uno de estos valores intangibles, la responsabilidad social corporativa (RSC), que desde hace unos años está viendo incrementado su desarrollo en el ámbito empresarial. Y es que, a lo largo de los últimos años, se han dado diversos acontecimientos que han cambiado la manera de relacionarse entre empresa y sociedad. El fenómeno de la globalización, la creciente desconfianza de la ciudadanía hacia las organizaciones debido a los escándalos empresariales surgidos en las últimas décadas, el incremento de la sensibilidad social de la ciudadanía hacia el comportamiento de las corporaciones, la progresiva preocupación de la ciudadanía por el deterioro medioambiental y la evolución de las Tecnologías de la Información y la Comunicación protagonizado por el desarrollo de Internet como medio de comunicación masiva y facilitador de información para el consumidor, son algunos de los fenómenos que nos han llevado hacia el nuevo panorama empresarial en el que, a través de una adecuada RSC, las empresas están dando respuesta a las expectativas de la sociedad.

Aunque si hay un fenómeno clave para comprender el auge que está viviendo la RSC, ése es la redefinición de la empresa desde la teoría de los stakeholders de Freeman. Esta nueva manera de concebir las organizaciones parte de la idea de que maximizar solo el valor para los accionistas no es suficiente, existen otros actores clave en la organización (consumidores, empleados, proveedores, sociedad, etc.), que afectan y son afectados por ésta, y que por ende, se han de tener en cuenta. Hoy en día hablamos de prácticas de RSC por parte de las compañías dirigidas a todo el conjunto de sus stakeholders. Es así que, la redefinición de la empresa desde la teoría de los stakeholders, cuyos objetivos deben definirse directamente en relación con los intereses de sus stakeholders, se presenta como una de las principales razones del actual auge de RSC.

En esta investigación nos interesa centrar la discusión de la RSC en la identificación de los diferentes stakeholders de una empresa y cómo son tipificados. Tanto la identificación como su catalogación son aspectos que se derivan de la gestión estratégica de la RSC por parte de las empresas. Para una adecuada gestión estratégica de este valor intangible, que permita adecuar las prácticas de RSC a las necesidades de los stakeholders, las organizaciones deben determinar quiénes son sus diferentes grupos de stakeholders (Maignan et al., 2005; Marín, 2008; López, 2010; Rodríguez, 2013). Es por ello que el objetivo principal de este estudio es conocer cuáles son los principales stakeholders de una organización y cómo se catalogan.

Para delimitar el estudio hemos considerado pertinente analizar el caso de una empresa relevante en los sectores de la alimentación, el cuidado del hogar y la higiene personal, con un amplio reconocimiento a su RSC como es Unilever. Las preguntas de investigación derivadas del objetivo principal y concretadas en el estudio de caso analizado son:

Q1: En la RSC que realiza Unilever España, ¿cuáles son sus stakeholders principales? 
Q2: En la RSC que realiza Unilever España, ¿cómo son clasificados los stakeholders?

\section{Marco Teórico}

\subsection{Responsabilidad Social Corporativa}

El concepto de RSC se ha convertido en un factor clave en las agendas de todo tipo de empresas y ha visto incrementada su importancia en el ámbito académico (Sanclemente-Téllez, 2017), siendo abordado por diversos investigadores nacionales e internacionales, dando lugar a la existencia de diferentes definiciones sin consenso entre ellas (Villagra, 2015; Sanclemente-Téllez, 2017). No obstante, a este respecto, Barrio y Enrique (2015: 29), observan una serie de ítems que se repiten a la hora delimitar el concepto de RSC y presentan una definición que recoge los criterios presentes de manera más frecuente. De este modo definen RSC como «la consecuencia de un compromiso adoptado con todos sus grupos de interés en materia económica, social y medioambiental, con el objetivo de responsabilizarse de las consecuencias e impactos que derivan de sus acciones y maximizar la creación de valor compartido para todo el conjunto de stakeholders». Siendo el compromiso adoptado por parte de las empresas hacia sus stakeholders, uno de los pilares fundamentales que definen el propio concepto de RSC.

En este sentido, cabe destacar los trabajos de Walton (1967), Van Marrewijk (2003), Hopkins (2004), Basil y Weber (2006), Castillo (2009) y Cuervo (2009) quienes destacan en sus definiciones de RSC, la relación presente entre la empresa y sus stakeholders, mientras que investigadores como Davis y Blosmstrom (1966) y Waldman et al. (2006) o la organización Forum Empresa (2017) inciden, cuando describen el concepto de RSC, en la satisfacción de las necesidades, intereses y/u objetivos de los stakehol- ders por parte de las compañías. Siguiendo esta premisa, académicos como Jones (1980), Cuesta y Valor (2003), Smith (2003), García-Marzá (2004), Maignan y Ferrell (2004), Otubanjo (2013) y López-Villalta (2016) van más allá en sus exposiciones y subrayan la idea de una responsabilidad, compromiso y/u obligación adoptado por las organizaciones hacia sus stakeholders. Así mismo, en los últimos años han sido diversos los investigadores que han abordado sus estudios de RSC desde la perspectiva de los stakeholders (Costa y Menichini, 2013; Yusoff, Mohamad y Darus, 2013; Fatma, Rahman y Khan, 2014; Kim, 2014; Trapp, 2014; Park y Ghauri, 2015; Scandelius y Cohen, 2016; Lim y Greenwod, 2017; Yongming y Yini, 2017).

En definitiva, una de las ideas fundamentales en la definición del propio concepto de RSC es el compromiso establecido por parte de las organizaciones para satisfacer las necesidades e intereses de todo el conjunto de sus stakeholders. De este modo, la integración de los intereses de los stakeholders en la compañía, se presenta como un factor clave e implícito en la RSC (Villagra, 2015). Y es que no se puede entender la RSC sin comprender el paradigma actual de empresa formada, no solo por los accionistas y socios, sino por todo un conjunto de stakeholders para los que se trata de maximizar la creación de valor compartido ${ }^{1}$.

\subsection{Stakeholders}

El concepto stakeholder aparece por primera vez en 1963, en un memorándum interno del Instituto de Investigación de Stanford, definido

\footnotetext{
1 En inglés shared value. Concepto acuñado por Porter y Kramer (2002, 2006 y 2011), definido como «las políticas y prácticas operativas que mejoran la competitividad de una empresa al mismo tiempo que avanzan en las condiciones económicas y sociales de las comunidades en las que opera» (Porter y Kramer, 2011, p. 6).
} 
como «aquellos grupos sin cuyo apoyo la organización dejaría de existir» e incluyendo, entre ellos a accionistas, trabajadores, clientes, proveedores, prestamistas y sociedad (Freeman, 1984, p. 31-32). Es importante, aclarar el significado y origen de esta palabra, stakeholder, que ha sido traducida al castellano como «participante» (Perdiguero, 2003) o grupo de interés (Villafañe, 2004). El término stakeholder, representa un juego de palabras cuyo origen de partida sería el vocablo shareholder o stockholder (accionista), el que posee una acción o participación. Así, siguiendo este juego de palabras, de la unión de los vocablos stake (interés) y holder (poseedor), nace el término stakeholder, que no describe otra cosa, que a aquel que posee un interés con la empresa, definido por Freeman (1984, p. 25) como «cualquier grupo o individuo que pueda afectar o ser afectado por el logro de los objetivos de la empresa.»

Con el desarrollo de la sociedad del conocimiento cambian las necesidades y expectativas de la ciudadanía, provocando la modificación radical de muchos de los supuestos organizativos e institucionales anteriores, transformando la manera de relacionarse de la gente con las empresas (Perdiguero, 2003, p. 159). Por lo que, la teoría de los stakeholders supone un nuevo esquema de empresa que tiene en cuenta a todos esos individuos vinculados de algún u otro modo con la organización. Por consiguiente, pasamos a un nuevo modelo de empresa complejo, definido por los equilibrios y relaciones entre la compañía y sus stakeholders. Este nuevo enfoque supone un sistema más complejo de empresa que el establecido en la teoría económica neoclásica, asignando a la dirección la obligación de gestionar en función de los intereses de todos los individuos afectados por la actividad empresarial (Perdiguero, 2003, p. 158). En otras palabras, las empresas ya no son consideradas «únicamente como un instrumento destinado a obtener beneficio económico, sino como un grupo humano cuya meta consiste en satisfacer intereses de muy diverso tipo» (Cortina, 1999, p. 103).

Para satisfacer esa diversidad de intereses, es necesario que las empresas determinen quiénes son sus stakeholders y qué necesidades tiene cada uno de ellos. En este sentido, en relación a la literatura que aborda la tipificación de los stakeholders, cabe destacar el trabajo de Mitchell, Agel y Wood (1997) quienes establecen tres criterios de clasificación de stakeholders: poder, legitimidad y urgencia. El poder hace referencia al grado en el que uno o varios stakeholders son capaces de influir en otros para tomar decisiones que no habrían tomado por su cuenta. La legitimidad se ocupa del grado de influencia moral o legal que tiene un stakeholder sobre el comportamiento de la empresa. Y por último, la urgencia se refiere al grado en que los stakeholders consideran importantes sus demandas hacia la organización. El establecimiento de estos criterios, permite tipificar los stakeholders en latentes (aquellos que poseen uno solo de los atributos), expectantes (aquellos que poseen dos atributos) y definitivos (aquellos que poseen los tres atributos).

Posteriormente otros autores han ampliado esta tipificación proponiendo nuevos criterios para clasificar a los stakeholders, entre los que Bajo (2015) destaca las siguientes propuestas:

- En función del grado de influencia en el desarrollo del negocio, diferenciando entre primarios (cuyos intereses están directamente relacionados con el destino de la empresa) y secundarios (aquellos que pueden influir de manera indirecta en una corporación y/o que son menos afectados de 
manera directa por las actividades de la organización).

- Por su representatividad, diferenciando entre stakeholders directos (quienes dialogan por sus propios intereses) e indirectos (quienes dialogan en calidad de interlocutores de terceros).

- Según el control sobre los recursos estratégicos.

- En relación a la posesión de conocimientos y habilidades críticas o útiles para la consecución de objetivos.

- Por la capacidad de control del entorno.

- Según su implicación en la aplicación de la estrategia, diferenciando entre stakeholders convergentes (cuya estrategia está alineada con la empresa) y divergentes (cuya postura es escasamente coincidente con la del negocio).
A su vez, además de estas clasificaciones recogidas por Bajo, en la literatura que aborda la tipificación de stakeholders, existen otras clasificaciones que presentan distintos criterios de catalogación. Estos mismos autores, también realizan sus propias contribuciones en relación a la identificación de los diferentes stakeholders de una compañía (véase tabla 1). Tras la exploración de las diferentes aportaciones, se desprende que los principales stakeholders de una organización son, por orden de aparición: empleados y clientes identificados por los nueve autores trabajados, seguidos por accionistas/inversores y proveedores con ocho menciones, posteriormente aparecen los entes reguladores/gobiernos con siete menciones, los competidores y los medios de comunicación con seis alusiones y, en último lugar, la comunidad mencionada por cinco autores.

\section{Tabla 1 • Identificación de stakeholders}

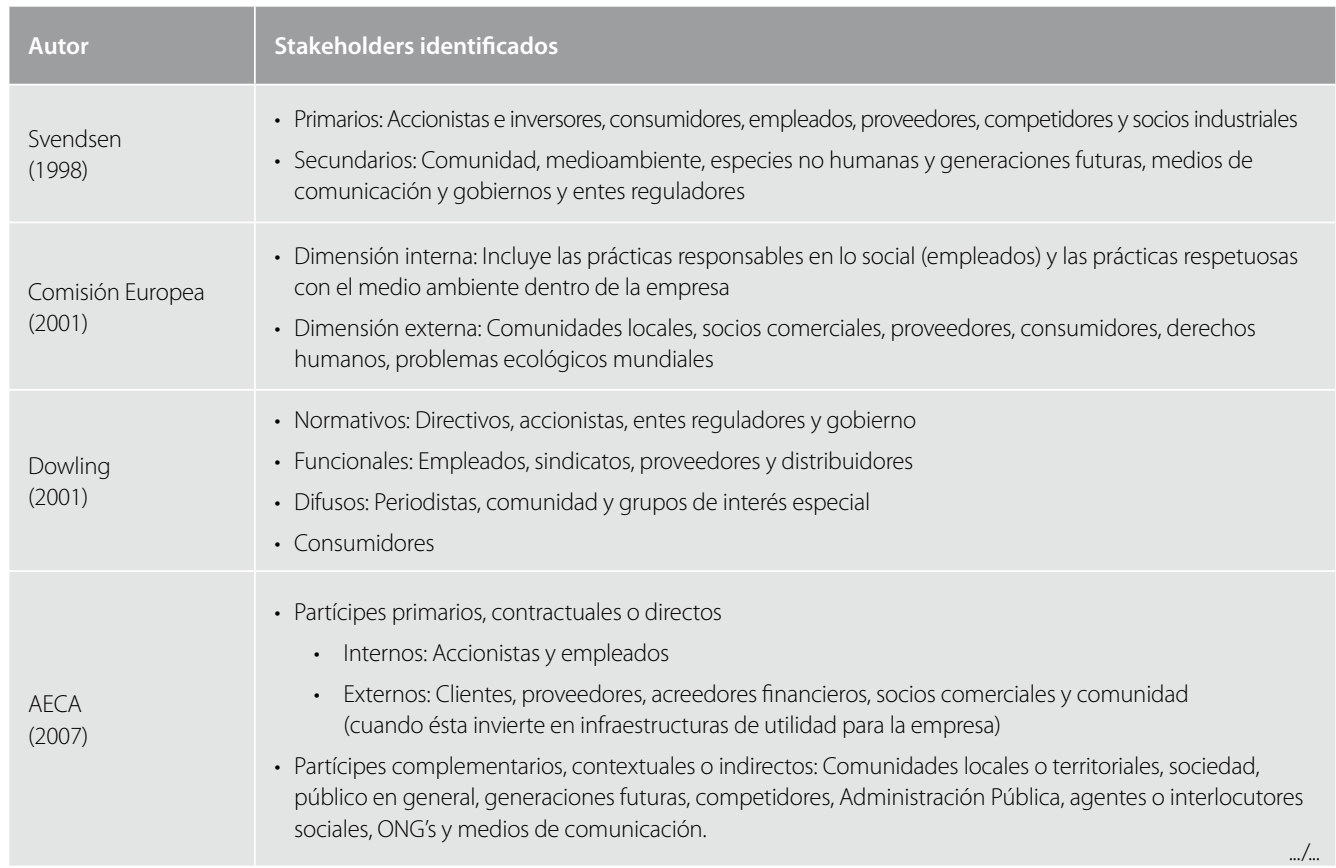




\begin{tabular}{|c|c|}
\hline Autor & Stakeholders identificados \\
\hline $\begin{array}{l}\text { Freeman, Harrison } \\
\text { y WicKs } \\
\text { (2008) }\end{array}$ & $\begin{array}{l}\text { - Primarios: Clientes, empleados, proveedores, financieros y comunidades } \\
\text { - Secundarios: Gobierno, competencia, grupos de los consumidores, medios de comunicación } \\
\text { y grupos de especial interés }\end{array}$ \\
\hline $\begin{array}{l}\text { Azuero } \\
(2009)\end{array}$ & $\begin{array}{l}\text { - Los que perderían algo si la empresa cerrara: Trabajadores y sus familias, consumidores, proveedores, } \\
\text { administraciones locales e inversores } \\
\text { - Los que pueden influir en la marca: Empleados, consumidores, medios de comunicación, ONG's, agencias } \\
\text { reguladoras y la competencia } \\
\text { - Grupos que representan intereses que pueden ser afectados por la operación de la empresa: Grupos } \\
\text { ecologistas, asociaciones cívicas, grupos de presión y gobiernos }\end{array}$ \\
\hline $\begin{array}{l}\text { Raghubir, Roberts, } \\
\text { Lemon y Winer } \\
\text { (2010) }\end{array}$ & $\begin{array}{l}\text { - Clásicos del marketing estratégico: consumidores, proveedores y otros colaboradores, competidores y la } \\
\text { empresa } \\
\text { - Internos: Inversores, empleados y directivos } \\
\text { - Externos: Sociedad, medio ambiente, entes reguladores, auditores y ONG's y medios de comunicación }\end{array}$ \\
\hline $\begin{array}{l}\text { Navarro } \\
(2012)\end{array}$ & $\begin{array}{l}\text { - Internos: Accionistas, socios, directivos, sindicatos, trabajadores y socios estratégicos } \\
\text { - Externos: Autoridades, grupos de presión, ONG's, competidores y consumidores }\end{array}$ \\
\hline Bajo (2015) & $\begin{array}{l}\text { - Primarios: Clientes, inversores, proveedores y empleados } \\
\text { - Secundarios: ONG's, administraciones públicas, medios de comunicación y grupos de presión }\end{array}$ \\
\hline
\end{tabular}

\section{Metodología}

Se ha planteado una investigación basada en el estudio de un caso real utilizando técnicas cualitativas. El método del estudio de caso se define como un tipo de investigación empírica que utiliza múltiples fuentes de evidencia para investigar un fenómeno contemporáneo en su contexto de vida real, sobre todo en aquellas situaciones en las que la frontera entre el fenómeno y el contexto no queda claramente delimitada (Yin, 2003, pp. 13-14). Este método de investigación es especialmente adecuado cuando el propósito de la investigación es comprender y explicar un fenómeno utilizando las fuentes de información que sean requeridas. En la presente investigación, no pretendemos conseguir una representación estadística, sino que, nuestra aspiración es obtener una generalización analítica «en la medida en que el caso es pertinente teóricamente (es decir, relevante), se puede relacionar las con- clusiones de un estudio con una teoría o conjunto de ellas» (Coller, 2005, p. 68).

El caso de estudio seleccionado ha sido Unilever España, una multinacional relevante en los sectores de la alimentación, el cuidado del hogar y la higiene personal ${ }^{2}$ y con un amplio reconocimiento de su RSC ${ }^{3}$, que cuenta con un extenso plan de RSC a nivel nacional permitiendo realizar un profundo análisis de su mapa de stakeholders en materia de RSC. A continuación se describen las diferentes iniciativas que conforman la RSC de Unilever España:

2 Unilever comercializa las marcas producto: Frigo, Flora, Knorr, Ligeresa, Calvé, Ben\&Jerry's, Tulipán, Lipton, Hellmann's, Maizena, Skip, Mimosín, Domestos, Cif, Dove, Rexona, Axe, Williams, Signal, Pond's, Timotei y Tresemmé.

3 Unilever ha sido galardonada por su RSC (Premio Nacional de Marketing a la Ética y la Sostenibilidad, CSR Online Awards y Gothenburg Award for SustainAbility Development) y ha liderado rankings de sostenibilidad (Tomorrow Value's Rating y Globescan/Sustainability) en los últimos años. 
- El Plan de Agricultura Sostenible de Knorr, con Agraz, un proveedor extremeño de tomate en polvo y concentrado. A través de acciones como la reducción de pesticidas y fertilizantes, el uso de sondas y tensiómetros para disminuir el consumo de agua, la rotación del suelo de cultivo, el reciclaje, la formación a los agricultores, acciones para proteger la biodiversidad (respetar las lindes de cultivo, instalación de cajas nido, creación de refugios para insectos y establecimiento de una charca para mamíferos y odonatos) y el cumplimiento de los once indicadores del código de agricultura sostenible de Unilever, la compañía busca mejorar la sostenibilidad y competitividad de su proveedor Agraz.

- El programa Soy Frigo, cuyo objetivo es reducir el paro juvenil en España a través de un modelo de negocio de venta de helados en espacios públicos.

\section{- El programa de salud cardiovascular de} Flora ProActiv, cuya finalidad es concienciar sobre la salud cardiovascular y ayudar a la población a reducir el colesterol, consta de las siguientes acciones:

- La plataforma científica Instituto Flora.

- La celebración del Día Nacional Contra el Colesterol (19 de septiembre), cuyo objetivo es educar sobre la reducción del colesterol a través de un estilo de vida saludable. Para ello, profesionales de la salud como la FEDN (Fundación Española de Dietistas y Nutricionistas) elaboran estudios sobre el impacto que tiene el colesterol elevado en la población y se realiza un acto en el centro de Madrid con diferentes actividades: mediciones de los niveles de colesterol, showcookings de alimentación saluda- ble, actividades físicas dirigidas y consejos nutricionales.

- El Reto ProActiv, que consiste en la reducción del colesterol en un periodo de 2-3 semanas a partir de un cambio de hábitos saludables.

- Un showcooking y un recetario con Flora ProActiv dirigidos a dietistas-nutricionistas.

- El Plan Unilever Comparte, que consiste en un conjunto de acciones de RSC dirigidas al municipio de Viladecans (Barcelona), donde se encuentra la sede de Unilever España. A continuación se exponen las diferentes acciones que conforman el Plan Unilever Comparte:

- Talleres Desayuno saludable pan con Tulipán e higiene bucal Signal sobre hábitos alimentarios para niños de primaria y talleres de Autoestima Dove a jóvenes de secundaria con el fin de hacerles ganar más confianza en sí mismos apostando por una belleza real.

- Concurso Canciones de 5 minutos para la ducha en el que las escuelas del municipio deben crear una canción para el momento del baño, con el fin fomentar la reducción del consumo de agua.

- Concurso Gran Estalvi dirigido a los vecinos del municipio para ver quién reduce más su consumo de agua, luz y gas.

- Caminata Solidaria cuyos fondos recaudados se destinan a la Fundación Viladecans Solidaria para ayudar en el pago de necesidades básicas a familias de la ciudad que lo necesiten.

- Cursos de cocina equilibrada y económica a familias de Viladecans desestructuradas y/o con pocos recursos. 
- Conferencias de los directivos de Unilever España dirigidas a las pymes del territorio y participación de la compañía en los encuentros entre pymes y grandes empresas que organiza el Ayuntamiento de Viladecans.

- Coaching de voluntarios de Unilever a jóvenes del municipio en riesgo de exclusión social a través de la Fundación Exit.

- Colaboración con servicios sociales, Cáritas y Cruz Roja en el reparto de alimentos y productos de necesidad básica a familias del municipio por debajo del umbral de la pobreza y colaboración con CAVIGA (un centro ocupacional para personas con disminución psíquica de Viladecans) y ASDIVI (una asociación para la integración de personas con discapacidad de Viladecans) realizando diferentes actividades de voluntariado.

- Programa de Voluntariado UNIdos en Viladecans.

- Diferentes acciones enfocadas a mejorar el bienestar de los empleados y el lugar de trabajo.

Para llevar a cabo el estudio de caso, el diseño de la investigación se ha basado en la técnica de la triangulación, que como señala Soler (2011, p.192) consiste en «contrastar la información (...) la acción de reunión y cruce dialéctico de toda la información pertinente al objeto de estudio surgida en una investigación». Para ello, se ha combinado la revisión de documentos escritos con la realización de doce entrevistas enfocadas a actores que forman parte de la gestión de la RSC de Unilever España (véase tabla 2).

\section{Tabla 2 • Fuentes de información de la investigación}

\begin{tabular}{|c|c|}
\hline \multirow{4}{*}{$\begin{array}{l}\text { Documentos escritos } \\
\text { revisados }\end{array}$} & Plan Unilever para una Vida Sostenible. \\
\hline & Plan Unilever Comparte: Guía de desarrollo de la comunidad. \\
\hline & Web de Unilever España. \\
\hline & Noticias sobre la RSC de Unilever España en medios de comunicación ${ }^{4}$. \\
\hline \multirow{4}{*}{$\begin{array}{l}\text { Agentes informantes } \\
\text { entrevistados }\end{array}$} & 7 agentes internos de Unilever España \\
\hline & $\begin{array}{l}\text { (La Directora de Comunicación y RSC, tres miembros del Departamento de Comunicación, un miem- } \\
\text { bro del Comité de Sostenibilidad, el project leader de la iniciativa de RSC «Soy Frigo» y la responsable } \\
\text { del Departamento de Nutrición y Salud en Unilever España) }\end{array}$ \\
\hline & 5 agentes externos a Unilever España \\
\hline & $\begin{array}{l}\text { (Dos empleados del departamento de sostenibilidad de Agraz, dos colaboradores del Ayuntamiento } \\
\text { de Viladecans y un miembro del patronato de la Fundación Española de Dietistas y Nutricionistas). }\end{array}$ \\
\hline
\end{tabular}

Con el fin de dar respuesta a las preguntas de investigación establecidas, se han planteado las siguientes categorías de análisis que han guiado la recolección de datos y la clasificación de la información:

4 Véase anexo 1 con el listado de las noticias y medios de comunicación revisados.
- Stakeholders de Unilever España en materia de RSC.

- Stakeholders de la iniciativa de RSC Plan de Agricultura Sostenible de Knorr.

- Stakeholders de la iniciativa de RSC Proyecto Soy Frigo. 
- Stakeholders de la iniciativa de RSC Programa de salud cardiovascular de Flora ProACtiv.

- Stakeholders de la iniciativa de RSC Plan Unilever Comparte.

- Stakeholders de la iniciativa de RSC de mejora del bienestar de los empleados.

- Clasificación de los stakeholders de Unilever España en materia de RSC.

- Clasificación de los stakeholders de la iniciativa de RSC Plan de Agricultura Sostenible de Knorr.

- Clasificación de los stakeholders de la iniciativa de RSC Proyecto Soy Frigo.

- Clasificación de los stakeholders de la iniciativa de RSC Programa de salud cardiovascular de Flora ProACtiv.

- Clasificación de los stakeholders de la iniciativa de RSC Plan Unilever Comparte.

- Clasificación de los stakeholders de la iniciativa de RSC de mejora del bienestar de los empleados.

La recogida de datos se realizó entre los meses de febrero y junio de 2015. Para la recogida de datos a través de las entrevistas enfocadas se elaboró un guión esquemático con los ítems a tratar con cada uno de los entrevistados. Posteriormente, el análisis de datos se ejecutó en dos etapas: una primera analizando el contenido de cada uno de los documentos revisados y las entrevistas realizadas de manera individual, y una segunda en la que se codificó el contenido de manera global en función de las categorías de análisis previamente expuestas. Para ello se empleó el software Maxqda12.

Durante el análisis de las entrevistas se detectó la participación de los medios de comunicación en la divulgación de las acciones de RSC de la compañía. Para corroborar este dato, se decidió realizar una revisión de los medios de comunicación que ofrecían noticias al respecto. Para ello, durante el mes de diciembre de 2015, se realizó una búsqueda en Google con las palabras Unilever y RSC/RSE/responsabilidad social. Además, se llevó a cabo una exploración, bajo los mismos parámetros, en los buscadores de las versiones digitales de los periódicos $\mathrm{ABC}, \mathrm{El}$ Mundo, El País, El Periódico, La Vanguardia, El Economista, Expansión, Compromiso RSE, Corresponsables y Diario Responsable.

\section{Resultados}

En referencia a la Q1, en la RSC que realiza Unilever España, los stakeholders que se han identificado son: consumidores, empleados, proveedores, distribuidores, administración pública, organizaciones sin ánimo de lucro, empresas colaboradoras, medios de comunicación, comunidad local y sociedad en general. Esta identificación de manera global de los stakeholders de la compañía, se ha realizado a partir de los stakeholders registrados en cada una de las iniciativas de RSC que realiza Unilever España (véase tabla 3).

De este modo, para el Plan de Agricultura Sostenible de Knorr, podemos afirmar que la compañía realiza diferentes acciones destinadas a su proveedor Agraz y a la sociedad en general, con el objetivo de mejorar la calidad de los agricultores y reducir el impacto medioambiental. Para ello, diferentes organizaciones sin ánimo de lucro (British Trust for Ornitology, SEO Birdlife, Defensa y Estudio del Medio Ambiente, Sigfito) y la empresa Control Union colaboran con Unilever en el Plan de Agricultura Sostenible de Knorr. Los medios de comunicación, conforman otro stakeholder de esta iniciativa, en tanto que participan en su divulgación para darla a conocer. 
En relación al Proyecto Soy Frigo, los stakeholders identificados han sido la sociedad en general que se ve beneficiada de la creación de los puestos de trabajo que proporciona esta iniciativa; la administración pública, representada por los ayuntamientos que facilitan las licencias municipales de venta en espacios públicos y a través de cuyas bolsas de empleo y servicios sociales se realiza la selección de candidatos para la venta de helados; las organizaciones sin ánimo de lucro Fundación Exit y FAEDEI y la empresa Manpower que colaboran en la ejecución de este proyecto; y por último, los medios de comunicación que participan en la divulgación de esta acción de RSC.

Los stakeholders de Unilever España para del Programa de salud cardiovascular de Flora ProActiv son los consumidores a quienes se apoya en la reducción del colesterol; la sociedad en general a quien se conciencia sobre la salud cardiovascular y el colesterol; organizaciones sin ánimo de lucro (Fundación Española de Dietistas y Nutricionistas, Sociedad Española de Médicos de Atención Primaria, Fundación Hipercolesterolemia Familiar) y distribuidores (supermercados y centros comerciales) que colaboran en el desarrollo de esta iniciativa; y medios de comunicación.

El Plan Unilever Comparte, por su parte, se dirige al stakeholder de la comunidad local, constituida por la localidad de Viladecans y formada por diferentes subgrupos de interés como estudiantes de primaria y secundaria, familias por debajo del umbral de la pobreza, familias desestructuradas, pymes y jóvenes en riesgo de exclusión social. También participan en el desarrollo de este plan, diversos stakeholders como la administración pública, representada por el ayuntamiento de Viladecans; organizaciones sin ánimo de lucro (Cruz Roja, Caritas, Centro ocupacional para personas con disminución psíquica de Viladecans, Asociación para la integración de personas con discapacidad de Viladecans, Fundación Exit); y medios de comunicación.

Finalmente, la compañía realiza una batería de acciones dirigidas a mejorar el bienestar de los empleados. Este stakeholder, se divide a su vez en empleados de oficina (oficina de Viladecans), empleados de fábrica (fábrica de Leioa -País Vasco-) y empleados externos que trabajan en la oficina.

\section{Tabla $3 \cdot$ Stakeholders de Unilever España}

\begin{tabular}{l|l}
\hline Iniciativas RSC & Stakeholders \\
\hline $\begin{array}{l}\text { Proveedor Agraz. A través del Plan de Agricultura Sostenible de Knorr, Unilever realiza diferentes acciones } \\
\text { destinadas a mejorar la calidad de los agricultores y a reducir el impacto medioambiental. } \\
\text { Sociedad en general. A través del Plan de Agricultura Sostenible de Knorr, Unilever realiza diferentes acciones } \\
\text { destinadas al cuidado del medioambiente. } \\
\text { Organización sin ánimo de lucro BTO (British Trust for Ornitology). Colabora en la elaboración del capítulo } \\
\text { de biodiversidad del código de agricultura sostenible de Unilever y pone en contacto al proveedor Agraz } \\
\text { con SEO Birdlife para que les asesore en los temas de biodiversidad. } \\
\text { Plan de Agricultura } \\
\text { Organización sin ánimo de lucro SEO Birdlife. Ayuda al proveedor Agraz en la implementación de los dos } \\
\text { proyectos de biodiversidad. } \\
\text { Organización sin ánimo de lucro DEMA (Defensa y Estudio del Medio Ambiente). Fabrica las cajas nido } \\
\text { instaladas por Agraz. } \\
\text { Organización sin ánimo de lucro Sigfito. Se encarga del reciclaje de los envases de los fertilizantes y los } \\
\text { pesticidas utilizados por el proveedor Agraz. } \\
\text { Empresa colaboradora Control Union. Consultora contratada por Unilever para realizar la formación y } \\
\text { asesoría de las prácticas de cultivo sostenible necesarias para el cumplimiento del código de agricultura } \\
\text { sostenible por parte de los proveedores. } \\
\text { Medios de comunicación. Participan en la divulgación de las diferentes acciones de RSC. }\end{array}$ \\
\hline M......
\end{tabular}




\section{Stakeholders}

Iniciativas RSC

Proyecto Soy Frigo

Programa de salud cardiovascular de Flora ProActiv
Sociedad en general. A través del Proyecto Soy Frigo, Unilever fomenta la creación de puestos de trabajo de venta de helados.

Ayuntamientos. Facilitadores de las licencias municipales de venta en espacios públicos y a través de cuyas bolsas de empleo y servicios sociales se realiza la selección de candidatos para la venta de helados.

Empresa colaboradora Manpower. Consultora de recursos humanos encargada de la contratación de los jóvenes y la organización del curso de capacitación previo al inicio de la venta de helados.

Organización sin ánimo de lucro Fundación Exit. Fundación que trabaja con jóvenes en riesgo de exclusión social y colabora con Unilever en la selección de candidatos para la venta de helados.

Organización sin ánimo de lucro FAEDEI (federación de empresas de inserción en España). En el momento de la investigación, Unilever está realizando un convenio para trabajar con ellos las candidaturas y contrataciones de la edición de 2015.

Medios de comunicación. Participan en la divulgación de las diferentes acciones de RSC.

Consumidores. Mediante el Programa de salud cardiovascular de Flora ProActiv, Unilever ayuda a la reducción del colesterol de sus consumidores.

Sociedad en general. Mediante el Programa de salud cardiovascular de Flora ProActiv, Unilever conciencia sobre la salud cardiovascular y el colesterol.

Organización sin ánimo de lucro FEDN (Fundación Española de Dietistas y Nutricionistas). Elabora el estudio sobre el impacto que el colesterol elevado tiene en la sociedad y participa como portavoz científico el Día Nacional contra el Colesterol.

Organización sin ánimo de lucro SEMERGEN (Sociedad Española de Médicos de Atención Primaria). Participa como portavoz científico presentando el estudio demoscópico que elabora para el Día Nacional contra el Colesterol.

Organización sin ánimo de lucro Fundación Hipercolesterolemia Familiar. Participa en la edición del Día Nacional contra el Colesterol de 2015.

Distribuidores. Durante el Día Nacional Contra el Colesterol centros comerciales y supermercados colaboran ofreciendo sus instalaciones para la realización de las mediciones de colesterol a la población.

Medios de comunicación. Participan en la divulgación de las diferentes acciones de RSC.

Comunidad local. A través del Plan Unilever Comparte, Unilever trabaja en la mejora del medioambiente, la salud, el bienestar y la calidad de vida de los habitantes de la localidad de Viladecans: estudiantes de primaria y secundaria, familias por debajo del umbral de la pobreza, familias desestructuradas, pymes y jóvenes en riesgo de exclusión social.

Ayuntamiento de Viladecans. Diferentes áreas del ayuntamiento participan en el desarrollo de las acciones que conforman el Plan Unilever Comparte: Departamento de Comunicación, Área de Servicios a Empresas, Área de Educación, Área de Medioambiente, Área de Infraestructuras, Servicios Sociales, Fundación Viladecans Solidaria, centros educativos.

Organización sin ánimo de lucro Cruz Roja. Participa en la gestión del reparto de alimentos y productos de necesidad básica a familias del municipio por debajo del umbral de la pobreza. También colabora en la organización de la Caminata Solidaria.

Plan Unilever

Organización sin ánimo de lucro Caritas. Participa en la gestión del reparto de alimentos y productos de necesidad básica.

Organización sin ánimo de lucro CAVIGA (Centro ocupacional para personas con disminución psíquica de Viladecans). Con quien colabora Unilever realizando actividades de voluntariado para sus usuarios.

Organización sin ánimo de lucro ASDIVI (Asociación para la integración de personas con discapacidad de Viladecans). Con quien colabora Unilever realizando actividades de voluntariado para sus usuarios.

Organización sin ánimo de lucro Fundación Exit. A través de esta fundación, voluntarios de Unilever realizan coaching a jóvenes del municipio en riesgo de exclusión social.

Medios de comunicación. Participan en la divulgación de las diferentes acciones de RSC. 


\begin{tabular}{l|l}
\hline \multicolumn{1}{|c|}{ Iniciativas RSC } & \multicolumn{1}{c}{ Stakeholders } \\
\hline $\begin{array}{l}\text { Mejora del bienestar } \\
\text { de los empleados }\end{array}$ & $\begin{array}{l}\text { Empleados. Unilever lleva a cabo acciones orientadas a mejorar el bienestar de los empleados y el lugar de } \\
\text { trabajo. Este stakeholder se divide en: empleados de oficina, empleados de fábrica, empleados externos que } \\
\text { trabajina. }\end{array}$ \\
\hline & Medios de comunicación. Participan en la divulgación de las diferentes acciones de RSC. \\
\end{tabular}

Con respecto a la Q2, para Unilever España, los partnerships con terceros tienen una gran relevancia para llevar a cabo sus acciones de RSC, por lo que la compañía clasifica sus stakeholders en función de si son los destinatarios de sus acciones de RSC o si son partícipes del desarrollo de las diferentes acciones de RSC (véase tabla 4). De este modo, los stakeholders de Unilever España son catalogados en beneficiarios y partners. Los primeros hacen referencia a aquellos stakeholders a los que van dirigidas sus acciones de RSC y están formados por los consumidores, los empleados, los proveedores (Agraz), la comunidad local (municipio de Viladecans) y la sociedad en general. Los segundos corresponden a aquellos que colaboran con Unilever para que las diferentes acciones de RSC se lleven a cabo. Éstos últimos están formados por organizaciones sin ánimo de lucro (BTO, SEO Birdlife, DEMA y Sigfito que intervienen en la aplicación española del Plan de Agricultura Sostenible de Knorr; Fundación
Exit y FAEDEI que participan en el Proyecto Soy Frigo; FEDN, SEMERGEN, Fundación Hipercolesterolemia Familiar que colaboran en el programa de Salud Cardiovascular de Flora ProActiv; y Cruz Roja, Caritas, CAVIGA, ASDIVI y Fundación Exit que están implicadas en el Plan Unilever Comparte); empresas colaboradoras (la consultora de recursos humanos Manpower que interviene en la contratación y formación del Proyecto Soy Frigo y la consultora Control Union que asesora al proveedor Agraz en las prácticas de cultivo sostenible); administración pública (representada por el ayuntamiento de Viladecans y los ayuntamientos en cuyos municipios se lleva a cabo el proyecto Soy Frigo); distribuidores (centros comerciales y supermercados en cuyas áreas se realizan las mediciones de colesterol durante el Día Nacional Contra el Colesterol); y medios de comunicación que participan en la divulgación de las acciones de RSC de la compañía a través de la publicación de noticias al respecto.

\section{Tabla 4 - Clasificación de stakeholders de Unilever España}

\begin{tabular}{|l|l|l|}
\hline Iniciativas RSC & $\begin{array}{l}\text { Clasificación de } \\
\text { stakeholders }\end{array}$ & Stakeholders \\
\hline Beneficiarios & $\begin{array}{l}\text { Proveedor Agraz. } \\
\text { Sociedad en general. }\end{array}$ \\
\hline $\begin{array}{l}\text { Plan de Agricultura } \\
\text { Sostenible de Knorr }\end{array}$ & Partners & $\begin{array}{l}\text { Organizaciones sin ánimo de lucro: BTO (British Trust for Ornitology), } \\
\text { Organización sin ánimo de lucro SEO Birdlife, DEMA (Defensa y Estudio } \\
\text { del Medio Ambiente), Sigfito. }\end{array}$ \\
\hline $\begin{array}{l}\text { Empresa colaboradora Control Union. } \\
\text { Medios de comunicación. }\end{array}$
\end{tabular}




\begin{tabular}{|c|c|c|}
\hline Iniciativas RSC & $\begin{array}{l}\text { Clasificación de } \\
\text { stakeholders }\end{array}$ & Stakeholders \\
\hline \multirow[b]{2}{*}{ Proyecto Soy Frigo } & Beneficiarios & Sociedad en general. \\
\hline & Partners & $\begin{array}{l}\text { Administración pública: Ayuntamientos. } \\
\text { Empresa colaboradora Manpower. } \\
\text { Organizaciones sin ánimo de lucro: Fundación Exit, FAEDEI (federación } \\
\text { de empresas de inserción en España). } \\
\text { Medios de comunicación. }\end{array}$ \\
\hline \multirow[b]{2}{*}{$\begin{array}{l}\text { Programa de salud } \\
\text { cardiovascular de } \\
\text { Flora ProActiv }\end{array}$} & Beneficiarios & $\begin{array}{l}\text { Consumidores. } \\
\text { Sociedad en general. }\end{array}$ \\
\hline & Partners & $\begin{array}{l}\text { Organizaciones sin ánimo de lucro: FEDN (Fundación Española de Dietistas } \\
\text { y Nutricionistas), SEMERGEN (Sociedad Española de Médicos de Atención } \\
\text { Primaria), Fundación Hipercolesterolemia Familiar. } \\
\text { Distribuidores. } \\
\text { Medios de comunicación. }\end{array}$ \\
\hline \multirow[b]{2}{*}{$\begin{array}{l}\text { Plan Unilever } \\
\text { Comparte }\end{array}$} & Beneficiarios & Comunidad local. \\
\hline & Partners & $\begin{array}{l}\text { Administración pública: Ayuntamiento de Viladecans. } \\
\text { Organizaciones sin ánimo de lucro: Cruz Roja, Caritas, CAVIGA, ASDIVI, } \\
\text { Fundación Exit. } \\
\text { Medios de comunicación. }\end{array}$ \\
\hline \multirow{2}{*}{$\begin{array}{l}\text { Mejora del bienestar } \\
\text { de los empleados }\end{array}$} & Beneficiarios & Empleados. \\
\hline & Partners & Medios de comunicación. \\
\hline
\end{tabular}

\section{Conclusiones}

A través de esta investigación se ha conseguido avanzar en la identificación y tipificación de los stakeholders de una empresa en materia de RSC. Gracias a la presente investigación, sabemos que, en la RSC que realiza Unilever España, los stakeholders establecidos por la compañía son los consumidores, empleados, proveedores, distribuidores, administración pública, organizaciones sin ánimo de lucro, empresas colaboradoras, medios de comunicación, comunidad local y sociedad en general. En cuanto a los accionistas/ inversores y competencia, señalados por algunos de los académicos trabajados en la revisión de la literatura, no hemos encontrado ninguna referencia hacia ellos en las diferentes acciones de RSC que realiza la compañía. En el caso de los accionistas, se debe a que la compañía considera la entrega de resultados a éstos como parte de su praxis empresarial, por lo que, el actuar de manera responsable con este público forma parte de la propia actividad de la empresa y queda fuera de las acciones de RSC de la compañía.

Además de esta identificación, el estudio llevado a cabo nos ha permitido conocer que Unilever no cataloga a sus stakeholders siguiendo los diferentes criterios expuestos en el marco teórico, sino que los clasifica en beneficiarios y partners. Los primeros se refieren a aquellos a los que la compañía dirige sus acciones de RSC (consumidores, empleados, proveedores, comunidad local y sociedad en general). Los segundos corresponden a un grupo de stakeholders a los que la organización no enfoca sus acciones de RSC, pero sí que colaboran con la empresa para que las diferentes acciones se lleven a cabo, de 
modo que se genera una relación win-win entre la compañía y los partners. En el caso analizado, éstos últimos están compuestos por organizaciones sin ánimo de lucro, empresas colaboradoras, administración pública, distribuidores y medios de comunicación. Véase tabla 5.

\section{Tabla 5. Mapa de stakeholders de Unilever España en materia de RSC}

\begin{tabular}{|l|l|}
\hline Clasificación & Stakeholders \\
\hline Beneficiarios & $\begin{array}{l}\text { Consumidores } \\
\text { Empleados } \\
\text { Proveedores } \\
\text { Comunidad local } \\
\text { Sociedad en general }\end{array}$ \\
\hline Partners & $\begin{array}{l}\text { Organizaciones sin ánimo de lucro } \\
\text { Empresas colaboradoras } \\
\text { Administración pública } \\
\text { Distribuidores } \\
\text { Medios de comunicación }\end{array}$ \\
\hline
\end{tabular}

Es interesante esta nueva clasificación de los stakeholders entre beneficiarios y partners. Recordemos que un stakeholder es «cualquier grupo o individuo que pueda afectar o ser afectado por el logro de los objetivos de la empresa» Freeman (1984, p. 25), de modo que, quedan incluidos tanto aquellos a los que se enfocan las iniciativas de RSC, y que por consiguiente se ven afectados por las acciones que realiza la empresa; como aquellos que participan en la ejecución de las acciones de RSC, afectando de este modo a la compañía y a su vez viéndose beneficiados ellos mismos, ya que los partners, a través de su colaboración con la compañía, ven como sus objetivos e intereses también son cumplidos.

Por consiguiente, la aportación de esta investigación, no se queda en la identificación y tipificación de los stakeholders de una organización, sino que va más allá, señalando la importancia de aquellos stakeholders denominados partners, sin cuya colaboración, no se podría dar la RSC de una empresa tal y como se está dando en la actualidad. Esta nueva clasificación permite a las organizaciones diferenciar entre dos grupos de stakeholders cuyo grado relacional con la organización en materia de RSC es diferente. De este modo, se distingue entre aquellos stakeholders que únicamente van a verse beneficiados de la RSC de la empresa, de aquellos con los que la empresa establece una relación de colaboración en la que el diálogo es más intenso.

Consideramos que esta investigación puede resultar un punto de partida para poder replicar el estudio en otras casuísticas y comprobar si estamos ante un caso particular a la hora de tipificar o clasificar a los stakeholders, o bien, encontramos una representatividad mayor de empresas que siguen este patrón, lo cual nos permitiría teorizar sobre esta cuestión. 


\section{Bibliografía}

AECA. (2007). Gobierno y Responsabilidad Social de la Empresa. Madrid: AECA

Azuero, D. (2009). La comunicación de la RSE. Propuestas para un modelo de comunicación responsable. Madrid: Forética Bajo, A. (2015). El diálogo empresa-stakeholders como fundamento del reconocimiento empresarial. En Comunicación y empresa responsable (pp. 91- 110). Barañáin: EUNSA.

Barrio, E. y Enrique, A.M. (2015). La Responsabilidad Social Corporativa en las empresas y su relación con los grupos de interés. Los casos de Unilever y Nestlé. Revista Internacional de Investigación en Comunicación aDResearch ESIC, $11(11), 26-39$.

Basil, D.Z. y Weber, D. (2006). Values motivation and concern for appearances: The effect of personality traits on responses to corporate social responsibility. International Journal of Nonprofit and Voluntary Sector Marketing, 11(1), 61-72.

Castillo, A. (2009). Relaciones Públicas. Teoría e historia. Barcelona: Editorial UOC

Coller, X. (2005). Estudio de casos. Madrid: Centro de Investigaciones Sociológicas

Comisión Europea. (2001). Libro Verde de la Comisión Europea para Fomentar un Marco Europeo para la Responsabilidad Social de las Empresas. Brussels.

Cortina, A. (1999). Ciudadanos del mundo. Hacia una teoría de la ciudadanía. Madrid: Alianza Editorial.

Costa, R. y Menichini, T. (2013). A multidimensional approach for CSR assessment: The importance of the stakeholder perception. Expert Systems with Applications, 40(1), 150-161.

Cuervo, A. (2009). Responsabilidad social corporativa, gobierno de la empresa y stakeholders. En Responsabilidad social: una reflexión global sobre la RSE (pp. 51-61). Madrid: Pretince Hall

Cuesta, M. y Valor, C. (2003). Responsabilidad social de la empresa: concepto, medición y desarrollo en España. Boletín Económico De ICE, Información Comercial Española, (2755), 7-20.

Davis, K. y Blomstrom, R.L. (1966). Business and its environment. New York: McGrawHill

Dowling, G. (2001). Creating corporate reputations. Identity, image, and performance. New York: Oxford University Press.
Fatma, M., Rahman, Z. y Khan, I. (2014). Multi-Item Stakeholder Based Scale to Measure CSR in the Banking Industry. International Strategic Management Review, 2(1), 9-20.

Forum Empresa. (2017). www.empresa.org/index.php? option $=$ com content $\&$ view $=$ article $\&$ id $=48 \&$ Itemid $=56$ Consultado el 25/11/2017.

Freeman, R.E. (1984). Strategic Management. A Stakeholder Approach. Boston: Pitman

Freeman, R.E., Harrison, J.S. y Wicks, A.C. (2008). Managing for Stakeholders: Survival, Reputation, and Success. New Haven, Connecticut: Yale University Press

García-Marzá, D. (2004). Ética empresarial. Del diálogo a la confianza. Madrid: Trotta

Hopkins, M. (2004). The Planetary Bargain. Corporate Social Responsibility Matters. Londres: Routledge.

Jones, T.M. (1980). Corporate social responsibility revisited, redefined. California Management Review, 22(3), 59-67.

Kim, Y. (2014). Strategic communication of corporate social responsibility (CSR): Effects of stated motives and corporate reputation on stakeholder responses. Public Relations Review, 40(5), 838-840.

Lim, J. S. y Greenwood, C. A. (2017). Communicating corporate social responsibility (CSR): Stakeholder responsiveness and engagement strategy to achieve CSR goals. Public Relations Review, 43, 768-776.

López, A. (2010). Observatorio global de intangibles. En La comunicación empresarial y la gestión de los intangibles en España y Latinoamérica. Informe Anual 2010 (pp. 13-37). Madrid: Pearson.

López-Villalta, J. (2016). La RSE: una vision empresarial que exige un impulse revitalizador. En Los condicionantes al desarrollo de la responsabilidad social empresarial. Memoria académica curso 2015-2016 (pp. 23-44). Madrid: Universidad Pontificia Comillas.

Maignan, I. y Ferrell, O.C. (2004). Corporate social responsibility and marketing: An integrative framework. Journal of the Academy of Marketing Science, 32(1), 3-19.

Maignan, I.; Ferrell, O. C. y Ferrell, L. (2005). A stakeholder model for implementing social responsibility in marketing. European Journal of Marketing, 39(9/10), 956-977. 
Marín, F. (2008). Responsabilidad social corporativa y comunicación. Madrid: Fragua.

Mitchell, R., Agle, B. y Wood, D. (1997). Toward a theory of stkeholder identification and salience: defending the principle of who and what really counts. Academy of Management Review, 22(4), 853-886.

Navarro, F. (2012). Responsabilidad Social Corporativa: Teoría y práctica. Madrid: Esic.

Otubanjo, O., 2013. Theorising the interconnectivity between corporate social responsibility (CSR) and corporate identity. Journal of Management and Sustainability. 3 (1), 74-94.

Park, B. I. y Ghauri, P. N. (2015). Determinants influencing CSR practices in small and medium sized MNE subsidiaries: A stakeholder perspective. Journal of World Business, 50(1), 192-204.

Perdiguero, T.G. (2003). La responsabilidad social de las empresas en un mundo global. Barcelona: Anagrama.

Porter, M. y Kramer, M. (2002). The competitive advantage to corporate philanthropy. Harvard Business Review, December, 5-16.

Porter, M. y Kramer, M. (2006). Strategy and Society: The link between competitive advantage and corporate social responsibility. Harvard Business Review, December, 1-13.

Porter, M. y Kramer, M. (2011). Creating Shared value. Harvard Business Review, January-February, 1-17.

Raghubir, P., Roberts, J., Lemon, K. y Winer, R. (2010). Why, when, and how should the effect of marketing be measured? A stakeholder perspective for corporate social responsibility metrics. Journal of Public Policy \& Marketing, 29 (1), 66-77.

Rodríguez, P. (2013). La Responsabilidad Social Corporativa en las Entidades Financieras de Depósito en España: Análisis desde el punto de vista de la información sobre stakeholders suministrada y sus determinantes. Tesis doctoral, Universidad de Córdoba. Sanclemente-Téllez, J.C. (2017). Marketing and Corporate Social Responsibility (CSR). Moving between broadening the concept of marketing and social factors as a marketing strategy. Spanish Journal of Marketing - ESIC, 21(S1), 4-25.
Smith, N.C. (2003). Corporate social responsibility: Whether or how? Centre for Marketing, 03-701, 1-37

Soler, P. (2011). La investigación cualitativa. Un enfoque integrador. En La investigación en comunicación. Métodos y técnicas en la era digital (pp. 189-236). Barcelona: Gedisa.

Scandelius, C. y Cohen G. (2016). Achieving collaboration with diverse stakeholders-The role of strategic ambiguity in CSR communication. Journal of Business Research, 69 (2016), 3487-3499.

Svendsen, A. (1998). The stakeholder strategy. San Francisco, California: Berrett-Koehler.

Trapp, N. L. (2014). Stakeholder involvement in CSR strategy-making? Clues from sixteen Danish companies. Public Relations Review, 40(1), 42-49.

Van Marrewijk, M. (2003). Concepts and definitions of CSR and corporate sustainability: Between agency and communion. Journal of Business Ethics, 44(2/3), 95-105.

Villafañe, J. (2004). La buena reputación. Madrid: Pirámide

Villagra, N. (2015). La gestión de intangibles y la Dirección de Comunicación. En Somos Estrategas (pp. 61-84). Barcelona: Gedisa.

Waldman, D.A., De Luque, M.S., Washburn, N. y House, R.J. (2006). Cultural and leadership predictors of corporate social responsibility values of top management: A GLOBE study of 15 countries. Journal of International Business Studies, 37(6), 823-837.

Walton, C.C. (1967). Corporate social responsibilities. Belmont, CA: Wadsworth

Yin, R. K. (2003). Case study research. Design and Methods. Thousand Oaks, California: Sage.

Yongming, Z. y Yini, S. (2017). The Impact of Coupling Interaction of Internal Control and CSR on Corporate Performance-Based on the Perspective of Stakeholder. Procedia Engineering, 174, 2017, 449-455.

Yusoff, H., Mohamad, S. S. y Darus, F. (2013). The Influence of CSR Disclosure Structure on Corporate Financial Performance: Evidence from Stakeholders' Perspectives. Procedia Economics and Finance, 7, 213-220. 


\section{Anexo}

\section{Anexo 1: Noticias sobre la RSC de Unilever España en medios de comunicación revisadas}

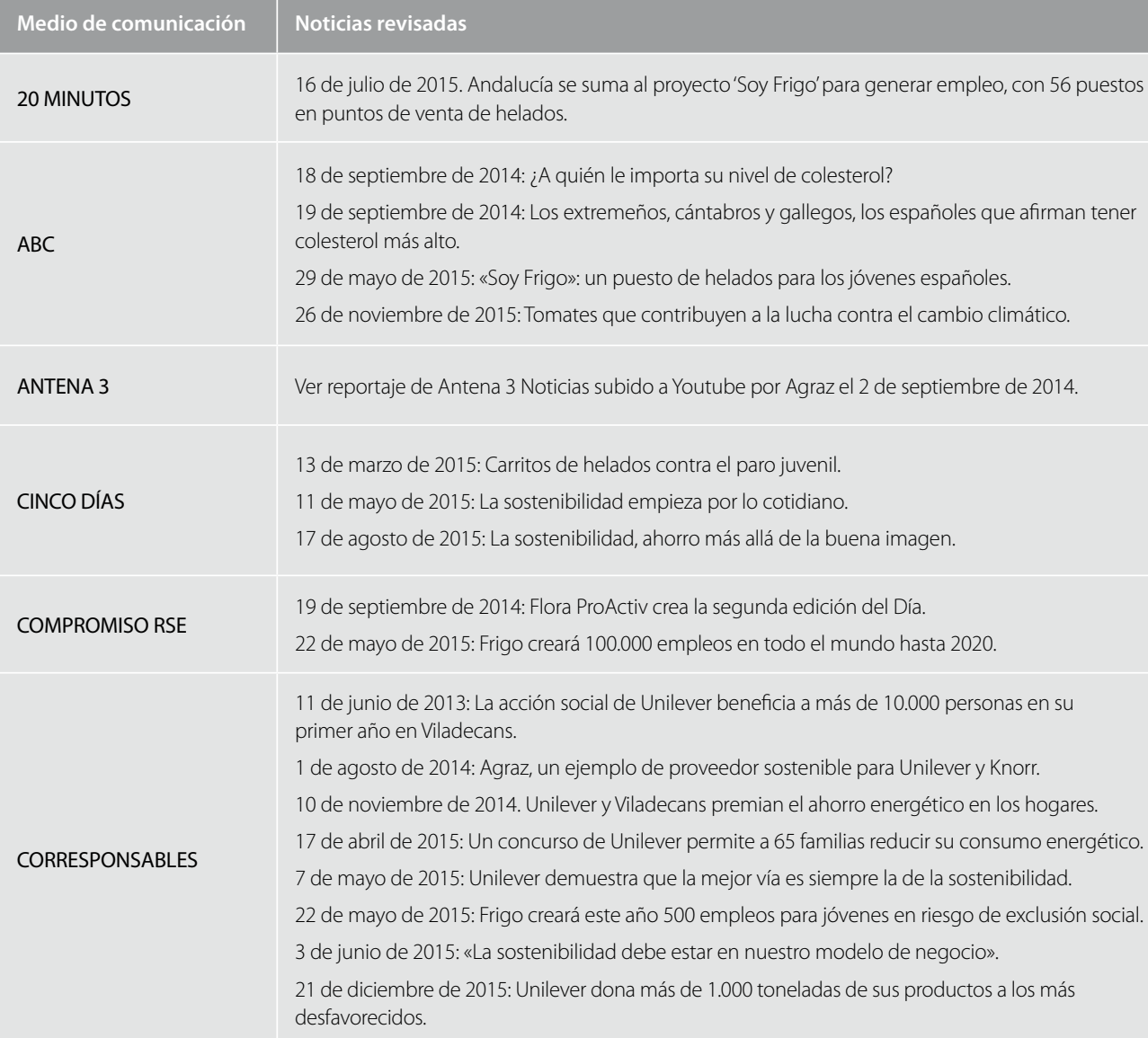

16 de julio de 2015. Andalucía se suma al proyecto 'Soy Frigo' para generar empleo, con 56 puestos en puntos de venta de helados.

18 de septiembre de 2014: ¿A quién le importa su nivel de colesterol?

19 de septiembre de 2014: Los extremeños, cántabros y gallegos, los españoles que afirman tener colesterol más alto.

29 de mayo de 2015: «Soy Frigo»: un puesto de helados para los jóvenes españoles.

26 de noviembre de 2015: Tomates que contribuyen a la lucha contra el cambio climático.

Ver reportaje de Antena 3 Noticias subido a Youtube por Agraz el 2 de septiembre de 2014.

13 de marzo de 2015: Carritos de helados contra el paro juvenil.

11 de mayo de 2015: La sostenibilidad empieza por lo cotidiano.

17 de agosto de 2015: La sostenibilidad, ahorro más allá de la buena imagen.

19 de septiembre de 2014: Flora ProActiv crea la segunda edición del Día.

22 de mayo de 2015: Frigo creará 100.000 empleos en todo el mundo hasta 2020.

11 de junio de 2013: La acción social de Unilever beneficia a más de 10.000 personas en su primer año en Viladecans.

1 de agosto de 2014: Agraz, un ejemplo de proveedor sostenible para Unilever y Knorr.

10 de noviembre de 2014. Unilever y Viladecans premian el ahorro energético en los hogares.

17 de abril de 2015: Un concurso de Unilever permite a 65 familias reducir su consumo energético.

7 de mayo de 2015: Unilever demuestra que la mejor vía es siempre la de la sostenibilidad.

22 de mayo de 2015: Frigo creará este año 500 empleos para jóvenes en riesgo de exclusión social.

3 de junio de 2015: «La sostenibilidad debe estar en nuestro modelo de negocio».

21 de diciembre de 2015: Unilever dona más de 1.000 toneladas de sus productos a los más desfavorecidos.

8 de mayo de 2014: Paul Polman, UNILEVER: «hemos aprendido que la sostenibilidad conduce al crecimiento del negocio».

31 de julio de 2014: La visión de Unilever es doblar el tamaño del negocio pero no a cualquier precio. 19 de septiembre de 2014: Colesterol, ese gran desconocido para un tercio de los españoles. 6 de mayo de 2015: Unilever: las marcas con propósito social crecen el doble.

22 de mayo de 2015: «Soy Frigo» creará 500 empleos en España y 100.000 en todo el mundo. 2 de julio de 2015: Balance del proyecto local «Unilever Comparte».

19 de septiembre de 2014: Un 20\% de los españoles que tienen el colesterol elevado no son conscientes de ello. Hoy se ha presentado la segunda edición del Día Nacional contra el Colesterol (DNC), que se celebrará mañana viernes en la Puerta del Sol de Madrid.

21 de mayo de 2015: Unilever lanza «Soy Frigo» para dar empleo a 100.000 jóvenes en todo el mundo. 
Medio de comunicación

\section{EL ECONOMISTA}

\section{EL MUNDO}

EL PAÍS

\section{EL PERIÓDICO}

\section{EXPANSIÓN}

\section{EXTREMADURA RADIO}

\section{LA VANGUARDIA}

ONDA CERO BADAJOZ

REVISTA DE VILADECANS

RTVE

\section{VILADECANSTV}

Noticias revisadas

6 de mayo de 2015: Unilever ayuda a 800.000 agricultores y logra que el 55\% de materias primas agrícolas procedan de fuentes sostenibles.

6 de mayo de 2015: Unilever ayuda a 800.000 agricultores y logra que el 55\% de materias primas agrícolas procedan de fuentes sostenibles.

14 de mayo de 2015: Dove organiza talleres de autoestima para adolescentes dentro del Plan Unilever para una vida Sostenible.

20 de mayo de 2015: Unilever: doblar ventas sin renunciar a la sostenibilidad.

22 de mayo de 2015: Frigo pone en marcha un plan para el fomento del empleo dirigido a jóvenes.

19 de octubre de 2015: El CEO mundial de Unilever, Paul Polman, afirma que la RSC de empresas es necesaria para el crecimiento sostenible.

18 de diciembre de 2015: Unilever España dona más de 1.000 toneladas de sus productos a colectivos en riesgo de exclusión social en 2015.

30 de julio de 2014: Gestionar bien datos mejora la calidad del tomate.

18 de septiembre de 2014: A uno de cada tres españoles no le preocupa su colesterol.

9 de junio de 2015: ¿Tienes menos de 30 años y estás buscando empleo? 500 puestos de trabajo te esperan.

18 de septiembre de 2014: Un tercio de los españoles no se preocupa de su nivel de colesterol sociedad

29 de octubre de 2015: Unilever convierte en una guía el aprendizaje del concurso «Gran Ahorro» de Viladecans.18 de diciembre de 2015: Unilever España dona más de 1.000 toneladas de sus productos a colectivos en riesgo de exclusión social en 2015.

26 de septiembre de 2013: Agraz anuncia su participación en un proyecto de tomates que ahorra 1,5 millones litros agua.

27 de septiembre de 2013: Entrevista a Víctor Rubio, director de sostenibilidad de Agraz www.youtube.com/watch?v=5bUtn9KT618

18 de septiembre de 2014: Los murcianos, entre los españoles más preocupados por su nivel de cholesterol.

11 de noviembre de 2014. Viladecans premia l'estalvi energètic a les cases.

16 de abril de 2015: Familias de Viladecans ahorran hasta 200 euros en cuatro meses en energía.

2 de junio de 2015: Viladecans organitza una caminada solidària per ajudar els més necessitats.

1 de julio de 2015: Unilever imparte talleres de nutrición y autoestima a 1.500 estudiantes de Viladecans.

3 de diciembre de 2015: Knorr se compromete a la reducción de emisiones de CO2 con su programa de agricultura sostenible.

18 de diciembre de 2015: Unilever España dona más de 1.000 toneladas de sus productos a colectivos en riesgo de exclusión social en 2015.

27 de septiembre de 2013: Entrevista a Víctor Rubio, director de sostenibilidad de Agraz www.youtube.com/watch?v=6xy8PWL92gc

Marzo de 2015: El concurso de ahorro energético en hogares El Gran Ahorro empieza a obtener resultados.

Mayo de 2015: Comunitats veïnals estalviadores. El Gran Estalvi premia el veïnat, que també ha reduït la seva factura.

Julio de 2015: 8a Caminada Solidària d'Unilever.

Ver reportaje de Agrosfera subido a Youtube por Agraz el 21 de octubre de 2013 www.youtube.com/watch?v=W58QjFcKbXk

17 de abril de 2015. Viladecans premia les comunitats de veïns que més energia han estalviat. 7 de junio de 2015: Viladecans es bolca amb la caminada solidària. 
